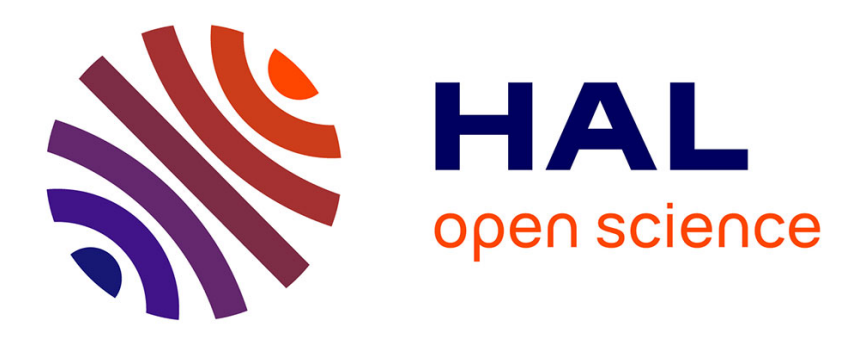

\title{
Adsorption of semi-dilute polymer solutions on fractal colloidal grains
}

\author{
C.M. Marques, J.-F. Joanny
}

\section{To cite this version:}

C.M. Marques, J.-F. Joanny. Adsorption of semi-dilute polymer solutions on fractal colloidal grains. Journal de Physique, 1988, 49 (7), pp.1103-1109. 10.1051/jphys:019880049070110300 . jpa-00210793

\section{HAL Id: jpa-00210793 https://hal.science/jpa-00210793}

Submitted on 1 Jan 1988

HAL is a multi-disciplinary open access archive for the deposit and dissemination of scientific research documents, whether they are published or not. The documents may come from teaching and research institutions in France or abroad, or from public or private research centers.
L'archive ouverte pluridisciplinaire HAL, est destinée au dépôt et à la diffusion de documents scientifiques de niveau recherche, publiés ou non, émanant des établissements d'enseignement et de recherche français ou étrangers, des laboratoires publics ou privés. 
Classification

Physics Abstracts

$61.25 \mathrm{H}-68.45 \mathrm{D}-82.65 \mathrm{M}-82.70 \mathrm{D}$

\title{
Adsorption of semi-dilute polymer solutions on fractal colloidal grains
}

\author{
C. M. Marques and J. F. Joanny \\ Ecole Normale Supérieure de Lyon, 46 Allée d'Italie, 69364 Cedex 07, France
}

(Reçu le 13 janvier 1988, accepté le 8 mars 1988)

\begin{abstract}
Résumé. - Nous étudions l'adsorption d'une solution semi-diluée de polymère sur des grains colloïdaux petits dont la surface a une géométrie fractale de dimension $D(5 / 3<D<3)$ en insistant sur le profil de concentration autour du grain et l'adsorbance qui représente le nombre total de monomères appartenant à des chaînes adsorbées. Sur des grains gros (surface plane), le profil de concentration a une structure self-similaire ; l'adsorbance est égale à l'excès de surface aux concentrations voisines de la concentration de recouvrement $\phi^{*}$ mais est beaucoup plus grande en solution concentrée, les chaînes adsorbées faisant de grandes boucles dans la solution. Sur des grains petits on trouve une assez grande variété de comportements d'échelle. Si la taille du grain est plus petite que la longueur de corrélation de la solution, le profil self-similaire s'étend jusqu'à une distance de l'ordre du rayon du grain, la concentration décroît ensuite plus rapidement vers sa valeur en volume. La géométrie de la surface détermine l'adsorbance aux faibles concentrations. Aux concentrations plus élevées la solution doit être considérée comme un fondu de blobs.
\end{abstract}

\begin{abstract}
We study the adsorption of a semidilute polymer solution on small colloidal grains with a fractal surface of dimension $D(5 / 3<D<3)$ focusing on the concentration profile around the grain and on the adsorbance which is the total number of monomers belonging to adsorbed chains. On large grains (flat surfaces) the concentration profile is self-similar. The adsorbance is equal to the surface excess at concentrations close to the overlap concentration $\phi^{*}$ but is larger in a concentrated solution, adsorbed chains making large loops in the solution. On small grains a rather large variety of scaling behavior is found. If the grain size is smaller than the solution correlation length, the self-similar profile extends up to distances of the order of the grain radius ; further away the concentration decays more rapidly towards its bulk value. The adsorbance is determined by the surface geometry in dilute solutions. At higher concentrations, the solution can be considered as a melt of blobs.
\end{abstract}

\section{Introduction.}

Most polymer solutions adsorb in a good solvent onto solid walls. The polymer chains form on the wall a fluffy layer which has been described in great details both experimentally [1] and theoretically [2]. The fluffy layer has a self-similar structure up to a length $\xi_{\mathrm{b}}$ of the order of the bulk correlation length of the solution which can be as large as a few hundred angströms (Fig. 1). This self-similar structure accounts well for many observed features of adsorbed polymer layers: surface excess, surface tension [3], hydrodynamic thickness, ellipsometric measurements [4], concentration profile as measured by neutron scattering [5]...

The existence of such diffuse polymer layers is of major importance in the study of colloidal stability. Colloidal particles may be coated with polymers and

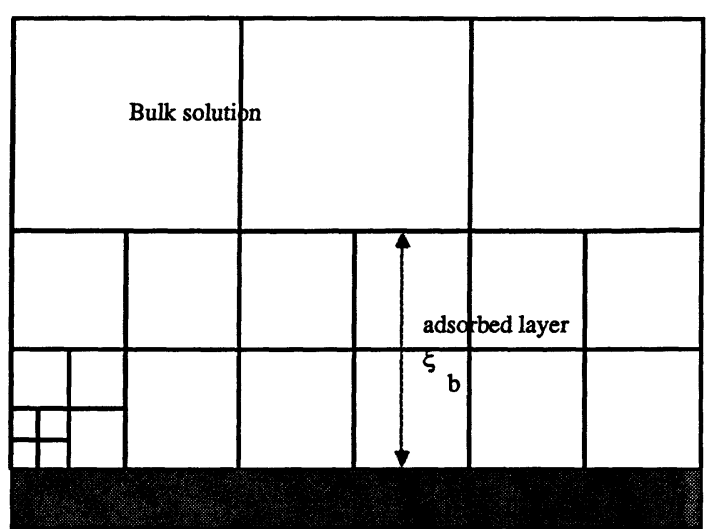

Fig. 1. - Self-similar structure of a semi-dilute solution in contact with a wall.

in a good solvent, the repulsive interaction between the adsorbed polymer layers eventually stabilizes the 
suspension. If the radius of the colloidal particles is much larger than the typical length scale of the solution (the correlation length $\xi_{\mathrm{b}}$ in a semi-dilute solution or the chain radius in a dilute solution) the description of the adsorbed layer is the same as on a plane (self-similar structure). We are interested here in the reverse limit where the radius $b$ of the particles is much smaller than the correlation length of the polymer solution (we will limit the study to semi-dilute solutions although some of the results may be used in a dilute solution as well). This has little application for colloidal stability but may be of some interest in the interpretation of experiments where a semi-dilute polymer solution adsorbs onto small surfactant micelles [6], it also gives a better understanding of the geometry of adsorbed polymer layers. We will characterize the polymer layer by the concentration around the particle $\phi$ and its adsorbance $\Gamma$. Close to the surface we distinguish between adsorbed chains which have at least one monomer in contact with the solid surface (or within a distance $a$ of the order of the monomer size) and non adsorbed chains. The adsorbance is the total number of monomers per unit surface belonging to adsorbed chains. In a dilute solution it is equal to the surface excess $\Gamma_{\mathrm{ex}}=\int \mathrm{d} \mathbf{r}\left(\phi-\phi_{\mathrm{b}}\right)$ where $\phi_{\mathrm{b}}$ is the bulk concentration away from the particle. In a more concentrated solution, the two quantities are different and $\Gamma>\Gamma_{\mathrm{ex}}$. Experimentally, for a polymer solution adsorbed on a colloidal suspension, $\Gamma$ may be measured by extracting the colloid and titrating the remaining liquid (assuming that the adsorption is irreversible). The knowledge of the adsorbance might also be of importance in the study of the adhesion of polymers on solid surfaces.

Our approach follows the scaling theory of De Gennes and Pincus [8]. We limit the study to a strong adsorption limit where the adsorption energy per monomer is of order $k T$. We thus ignore all the subtle tricritical effects related to the so-called special transition [7].

The paper is organized as follows. In section 2 we briefly review the important results concerning the adsorption of semi-dilute solutions on planar surfaces and discuss in details the adsorbance. This leads to a description of the adsorbed layer in terms of trains tails and loops as in the classical theories but the trains have here a slightly different meaning. These results are then extended to large fractal surfaces with a known fractal dimension $D$. In section 3 we study the adsorption on a small spherical grain and distinguish several regimes according to the relative value of the grain radius $b$ and the characteristic lengths of the solution, the chain radius $R$ and the correlation length $\xi_{\mathrm{b}}$; we then discuss fractal colloidal grains with a small size $b$. Section 4 presents our conclusions.

\section{Adsorption of a semi-dilute solution on a large colloidal grain.}

2.1 AdSORPTION ON A SOLID PLANE. - The concentration profile of a semi-dilute polymer solution close to a wall is obtained by minimization of the grand canonical free energy [8] :

$$
\Omega=\Omega_{\mathrm{S}}\left(\phi_{\mathrm{S}}\right)+\int\left\{f(\phi)-\mu_{\mathrm{ex}} \phi+\pi_{\mathrm{ex}}\right\} \mathrm{d} \mathbf{r} .
$$

Here $\Omega_{\mathrm{S}}$ is the surface energy which depends on the surface concentration $\phi_{\mathrm{S}}$. The free energy density $f(\phi)$ is given by [9]

$$
f(\phi)=\alpha \frac{T}{\xi^{3}}\left\{1+\left(m \frac{\xi(\phi)}{\phi} \nabla \phi\right)^{2}\right\} .
$$

$m$ and $\alpha$ are numerical constants of order unity ; the local correlation length $\xi(\phi)$ scales as $\phi^{-3 / 4}$.

The external chemical potential $\mu_{\mathrm{ex}}$ and osmotic pressure $\pi_{\mathrm{ex}}$ are imposed by the solution in the bulk of concentration $\phi_{\mathrm{b}}$ far from the surface

$$
\mu_{\mathrm{ex}} \sim \frac{T}{\xi_{\mathrm{b}}^{3} \phi_{\mathrm{b}}} \quad \pi_{\mathrm{ex}} \sim \frac{T}{\xi_{\mathrm{b}}^{3}}
$$

It is convenient here to caracterize the polymer solution by the order parameter $\psi$ defined as $\psi=\left(\phi a^{3}\right)^{3 / 8}$. The Euler-Lagrange equation minimizing the grand canonical free energy (1) is

$$
m_{1}^{2} \nabla^{2} \psi=\psi^{5}-\psi^{5 / 3} \psi_{\mathrm{b}}^{10 / 3}
$$

$m_{1}$ is a length proportional to the monomer length $a$ and the external chemical potential $\mu_{\mathrm{ex}}$ has been expressed as a function of the order parameter in the bulk $\psi_{\mathrm{b}}$. If $\psi$ does not vary in space, it is equal to $\psi_{\mathrm{b}}$.

When we study the adsorption on a plane, the concentration is a function only of the distance from the surface $z$ and equation (4) leads to the self similar profile of figure 1

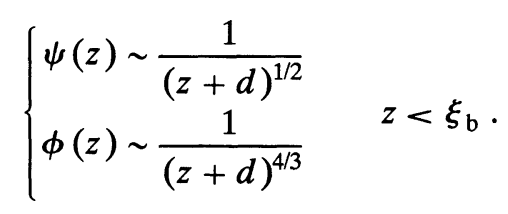

The interpolation length $d$ is obtained by minimization of the free energy with respect to the surface concentration $\phi_{\mathrm{S}}$; in situations of strong adsorption it is of the order of a monomer length $a$. At distances larger than $\xi_{b}$ the concentration relaxes exponentially towards its bulk value.

The surface excess $\Gamma^{\mathrm{ex}}$ is directly calculated from the concentration profile

$$
\Gamma^{\mathrm{ex}} \sim 1 / a^{2} .
$$

The adsorbance $\Gamma$ however cannot be directly 
obtained from the concentration profile, the description of a semi-dilute solution by equation (1) essentially assumes that the chains are infinite and does not distinguish between adsorbed and non adsorbed chains. An upper bound for $\Gamma$ is readily obtained by integrating the concentration between the wall and the radius of gyration of the chains in a semi-dilute solution $R\left(\phi_{\mathrm{b}}\right) \sim N^{1 / 2} \phi_{\mathrm{b}}^{-1 / 8}$

$$
\Gamma \leqslant \Gamma^{\mathrm{ex}}+\phi_{\mathrm{b}} R\left(\phi_{\mathrm{b}}\right) .
$$

In any case, $\Gamma$ is larger than $\Gamma^{\mathrm{ex}}$, we now argue that $\Gamma$ is also larger than the second term on the right hand side of (6). For a semi-dilute solution in contact with a neutral wall (neither attractive not repulsive) or a weakly attractive wall (attraction energy much smaller than $T$ ), the concentration profile is essentially flat. As in a melt, a finite fraction of the chains within a distance $R\left(\phi_{\mathrm{b}}\right)$ from the surface touch the surface, the adsorbance $\Gamma$ is thus equal to $R\left(\phi_{\mathrm{b}}\right)$ $\phi_{\mathrm{b}}$ (the subtle effects related to the special transition are not important here). As the attraction of the wall is increased, the adsorbance must increase, we thus approximate the adsorbance by

$\Gamma \sim \Gamma^{\mathrm{ex}}+\phi_{\mathrm{b}} R\left(\phi_{\mathrm{b}}\right) \sim 1 / a^{2}\left[1+N^{1 / 2}\left(\phi_{\mathrm{b}} a^{3}\right)^{7 / 8}\right]$.

This leads us to distinguish between two different regimes: in a rather dilute solution the second contribution is small and as expected $\Gamma=\Gamma^{\mathrm{ex}}$, in a more concentrated solution the second term is dominant and the semi-dilute solution has a behaviour closer to that of a melt. The crossover between these two regimes occurs at a concentration $\phi_{2}$ much larger than the overlap concentration $\phi^{*}$

$$
\phi_{2} \sim a^{-3} N^{-4 / 7} .
$$

The adsorbance $\Gamma$ is closely related to the number of monomers per adsorbed chains on the solid surface $N_{\mathrm{S}}=N / \Gamma a^{2}$. In dilute solutions $\left(\phi<\phi_{2}\right) N_{\mathrm{S}} \sim N$ a result which has already been discussed in details by several authors [10]. In concentrated solutions $N_{\mathrm{S}}$ is much smaller than $N$ and has the same mass dependence as in a melt $N_{\mathrm{S}} \sim N^{1 / 2}\left(\phi_{\mathrm{b}} a^{3}\right)^{-7 / 8}$.

This allows to give a precise description of the geometry of a semi-dilute solution in contact with a wall. Close to the wall, there exists a region of size $\xi_{\mathrm{b}}$ which we call the adsorbed layer (Fig. 1) where the concentration has a self-similar structure and is significantly larger than the bulk concentration $\phi_{\mathrm{b}}$.

At the overlap concentration $\phi^{*}$ the thickness of this layer is equal to the chain radius and the adsorbed chains are trapped inside the adsorbed layer. On the contrary in a concentrated solution, the thickness of the adsorbed layer is $\xi_{b}$ much smaller than the chain radius $R\left(\phi_{\mathrm{b}}\right)$ and an adsorbed chain can be viewed as a succession of trains and loops, the trains being pieces of chains trapped in the adsorbed layer. The size of the trains can be estimated by considering the semi-dilute solution as a melt of blobs of size $\xi_{\mathrm{b}}$ each containing $g=\xi_{\mathrm{b}}^{5 / 3}$ monomers. A blob belonging to a train has $g_{\mathrm{S}} \sim g$ monomers in contact with the surface. On a surface $\xi_{\mathrm{b}}^{2}$, the surface concentration being unity, $\xi_{\mathrm{b}}^{2} / g_{\mathrm{S}} \sim g^{1 / 5}$ chains are in contact. The self-similar picture of figure 1 imposes that only a finite number of these of order 1 escape from the adsorbed layer i.e. each chain has a probability $g^{-1 / 5}$ to escape, leading to a number of monomers per train

$$
p \sim g^{6 / 5} \sim \xi_{\mathrm{b}}^{2} \sim \phi_{\mathrm{b}}^{-3 / 2} .
$$

Combining equations (7) and (9) we obtain the average number of loops per chain $n_{1} \sim(N / g)^{1 / 2}$.

A comparison between the size of the trains $p$ and the polymerization index $N$ defines a second crossover concentration $\phi_{1}\left(\phi^{*}<\phi_{1}<\phi_{2}\right)$

$$
\phi_{1} \sim a^{-3} N^{-2 / 3} \text {. }
$$

When the bulk concentration is smaller than $\phi_{1}$ most adsorbed chains are trapped in the adsorbed layer, although some of them escape in the solution they make few loops. While increasing the concentration between $\phi_{1}$ and $\phi_{2}$, loops are formed ; this broad crossover regime is not easy to study in details. Finally in a more concentrated solution $\phi_{\mathrm{b}}>\phi_{2}$ the behaviour of the solution is reminiscent of that of a melt with an adsorbed layer of size $\xi_{\mathrm{b}}$.

The existence of these two crossover concentrations is related to the finite size of the chain and as shown below to the difference between the fractal geometry [11] of the solid surface $(D=2)$ and that of the polymer chain $(D=5 / 3)$. Although their definition seems clear from a theoretical point of view, their experimental determination might be difficult ; many quantities such as the surface excess, the concentration profile or the layer thickness show no crossover at these concentrations. They could however be observed by a direct determination of the adsorbance or the number of monomers per adsorbed chain on the surface.

2.2 AdSORPTION ON A FRACTAL SURFACE. - We now generalize these results to a large fractal surface of fractal dimension $D(5 / 3<D<3)$ and of macroscopic size $b$, following the ideas of De Gennes [12]. The surface excess has been calculated in reference [12]

$$
\Gamma^{\mathrm{ex}}=\frac{1}{b^{D}} \int_{0}^{R} \phi(z) \mathrm{d} \Omega
$$


The concentration $\phi(z)$ at a distance $z$ from the surface is given by equation (5) and the accessible volume $\Omega$ is measured with a length scale $z$, $\Omega=b^{D} z^{3-D}$. This leads to a surface excess

$$
\Gamma^{\mathrm{ex}}=a^{-D} \text {. }
$$

Following the same lines as above, we determine the adsorbance

$$
\Gamma=\Gamma^{\mathrm{ex}}+\frac{\phi_{\mathrm{b}} \Omega^{\prime}}{b^{D}} .
$$

The volume $\Omega^{\prime}$, containing the adsorbed chains is measured here with a length scale equal to the chain radius

$$
\begin{aligned}
& \Gamma \sim \frac{1}{a^{D}}\left(1+\phi_{\mathrm{b}} R^{3-D}\right) \sim \\
& \sim \frac{1}{a^{D}}\left(1+\phi_{\mathrm{b}}^{(5+D) / 8} N^{(3-D) / 2}\right) .
\end{aligned}
$$

On a fractal surface, the crossover concentration $\phi_{2}$ is thus given by

$$
\phi_{2} \sim a^{-3} N^{-\beta}, \quad \frac{1}{\beta}=\frac{2}{3-D}-\frac{1}{4} .
$$

The number of monomers per train $p$ scales as $p \sim \xi_{\mathrm{b}}^{D}$ and the other crossover concentration is

$$
\phi_{1} \sim a^{-3} N^{-\alpha} \quad \alpha=\frac{4}{3 D} .
$$

The existence of two different crossover concentrations is related to the existence of several characteristic length scales. Although the polymer solution has only one characteristic length scale its correlation length $\xi_{\mathrm{b}}$, the presence of the fractal surface introduces another characteristic length $\lambda \sim N^{1 / D}$. Notice that when $D$ is equal to $5 / 3$ i.e. to the polymer fractal dimension, $\lambda$ is equal to the radius of gyration (at $\phi^{*}$ ) and the two concentrations are equal to the overlap concentration $\phi *$.

\section{Adsorption of semi-dilute polymer solutions on small colloidal grains.}

3.1 CONCENTRATION PROFILE AROUND A SMALL SPHERICAL PARTICLE. - The spherical particle has a radius $b$ smaller than the semi-dilute solution correlation length $\xi_{\mathrm{b}}$. The profile around it is given by equation (4) in a spherical geometry

$$
m^{\prime 2}\left[\frac{\mathrm{d}^{2} \psi}{\mathrm{d} r^{2}}+\frac{2}{r} \frac{\mathrm{d} \psi}{\mathrm{d} r}\right]=\psi^{5}-\psi^{5 / 3} \psi_{\mathrm{b}}^{10 / 3}
$$

This same equation has been studied in details by De Gennes [13] for the behaviour of a critical binary mixture close to a sphere. We only give here a qualitative discussion and refer to this work for a more precise treatment. Close to the sphere, at a distance $z=r-b$ from the surface smaller than the radius $b$, the curvature term is negligible in $\left(4^{\prime}\right)$ and the concentration profile is the same as for the adsorption on a plane given by equation (5). At larger distances, the right hand side is negligible in $\left(4^{\prime}\right)$ and the order parameter satisfies a Laplace equation so that $\psi \sim \frac{1}{r}$. A rough matching with the planar profile at $r=2 b$ yields

$$
\phi(r) \sim \frac{b^{4 / 3}}{r^{8 / 3}} \quad r \gg b .
$$

This is valid as soon as $\psi>\psi_{\mathrm{b}}$ or $r \ll \sqrt{b \xi_{\mathrm{b}}}$. Linearization around the value $\psi=\psi_{\mathrm{b}}$ shows that at larger distances the concentration relaxes exponentially towards its bulk value with a decay length proportional to $\xi_{\mathrm{b}}$.

These results find a simple interpretation in terms of the loop size distribution around the particle. The number of loops of size between $l$ and $l+\mathrm{d} l$ may be estimated by assuming that these loops contribute for a finite fraction of the monomers at a distance $l$ from the surface following the lines of reference [14]

$$
\begin{cases}S(l) \sim \frac{b^{2}}{l^{3}} & l<b \\ S(l) \sim \frac{b^{4 / 3}}{l^{7 / 3}} & b<l<\sqrt{b \xi_{\mathrm{b}}} .\end{cases}
$$

The total number of small loops (with a size smaller than $b$ ) is proportional to $\left(\frac{b}{a}\right)^{2}$, the total number of large loops (of size larger than $b$ ) is of order 1. The solution forms thus a fluffy layer around the particle. The self-similar structure however can only include loops smaller than the grain size $b$. Eventually one adsorbed chain may form a large loop but the two ends of this loop are constrained within a distance $b$; the probability of formation of large loops is very small in a good solvent but the distribution is broad and these large loops build up the concentration profile given by equation (15). At distances larger than $\sqrt{b \xi_{b}}$ most monomers belong to non-adsorbed chains.

The same picture can be used to study adsorption on a small fractal grain of size $b$ and fractal dimension $D$. Close to the grain surface, at distances smaller than $b$, a fluffy self similar structure is formed similar to that formed on an infinite surface. Further away from the particle, $(r \gg b)$ the precise geometry of the surface is no longer important, only its macroscopic size counts (it fixes the distance to which the ends of the large loops are constrained) and the variation of the concentration with the distance from the center of the grain is given by equation (15). 
3.2 ADSORBED CHAINS ON A SMALL GRAIN. Rather than the adsorbance $\Gamma$, we calculate the total number of adsorbed chains $\gamma=\frac{\Gamma b^{D}}{N}$. We use the same method as on large grains. The number of adsorbed chains is the sum of two contributions, a contribution $\gamma_{1}$ related to the surface excess that we estimate from the concentration profile and a contribution $\gamma_{2}$ that we estimate from the number of adsorbed chains in an equivalent melt of blobs. We distinguish two types of grains, very small grains for which $b<\xi_{\mathrm{b}}$ and small grains for which $\xi_{\mathrm{b}}<b<R\left(\phi_{\mathrm{b}}\right)$.

3.2.1 Very small grains $b<\xi_{\mathrm{b}}$. - For very small grains $\gamma$ is equal to $\gamma_{1}$. The concentration profile has been studied in the preceding section; both small loops and large loops contribute to $\gamma_{1}$, the contribution of large loops being at most one chain

$$
\gamma_{1}=\gamma=1+\frac{b^{D}}{N}
$$

There are thus two concentration regimes. When $\phi_{\mathrm{b}}$ is larger than the concentration $\phi_{1}$ defined by equation (14), there is only one adsorbed chain. When $\phi_{\mathrm{b}}$ is smaller than $\phi_{1}, \gamma=1$ if $b<\lambda \sim N^{1 / D}$ and $\gamma \sim \frac{b^{D}}{N}$ if $b>\lambda$.

If $b$ is small enough, a single chain (or a number of order one) is in contact with the colloidal grain, only a small piece of this chain containing $N^{*} \sim\left(\frac{b}{a}\right)^{D}$ monomers is adsorbed, the two large tails dangling in the solution. As $b$ is increased, the number of adsorbed monomers $N^{*}$ becomes larger than $N$ and several chains are needed to saturate the surface of the grain. These chains are trapped in the adsorbed layer except for a few large loops which build the concentration profile given by equation (15).

3.2.2 Small grains $\xi_{\mathrm{b}}<b<R\left(\phi_{\mathrm{b}}\right)$. - The concentration profile is the same as that close to an infinite surface and $\gamma_{1}=\frac{b^{D}}{N}$.

To estimate $\gamma_{2}$, we consider the solution as a melt of blobs of $g$ monomers of size $\xi_{b}\left(g^{3 / 5}=\xi_{b}\right)$, each chain containing $n=\frac{N}{g}$ blobs ; we distinguish three regimes :

(i) when $\frac{b}{\xi_{\mathrm{b}}}$ is small, different blobs in contact with the surface belong to different chains

$$
\gamma_{2}=\left(\frac{b}{\xi_{\mathrm{b}}}\right)^{D}
$$

In the volume of one chain there are however $n^{1 / 2}$ different chains, a small grain smaller than the chain radius cannot be in contact with more than these $n^{1 / 2}$ chains; equation (17) ceases thus to be valid when $\frac{b}{\xi_{\mathrm{b}}}<n^{1 /(2 D)}\left(\frac{N}{g}\right)^{1 /(2 D)}$.

If $\phi_{\mathrm{b}}<\phi_{1}, \gamma_{2}<\gamma_{1}$ and $\gamma \sim \frac{b^{D}}{N}$; the number of monomers per adsorbed chain on the surface is proportional to $N$.

$$
\text { If } \phi_{\mathrm{b}}>\phi_{1}, \gamma_{2}>\gamma_{1} \text { and } \gamma=\left(\frac{b}{\xi_{\mathrm{b}}}\right)^{D} ; \text { the }
$$

number of monomers per adsorbed chain on the surface $N_{\mathrm{S}}$ is equal to the number of monomers in a train $p=\left(\frac{\xi_{\mathrm{b}}}{a}\right)^{D}$ (Eq. (9)) ;

(ii) when $\xi_{\mathrm{b}}\left(\frac{N}{g}\right)^{1 /(2 D)}<b<R\left(\phi_{\mathrm{b}}\right)$ all the chains occupying the same volume are linked to the surface and $\gamma_{2}$ is independent of the grain radius

$$
\gamma_{2} \sim\left(\frac{N}{g}\right)^{1 / 2}
$$

If $\phi_{\mathrm{b}}<\frac{b^{8 D / 5}}{N^{12 / 5}}, \gamma_{2}<\gamma_{1}$ and $\gamma=\frac{b^{D}}{N} ; N_{\mathrm{S}}$ is proportional to $N$.

$$
\text { If } \phi_{\mathrm{b}}>\frac{b^{8 D / 5}}{N^{12 / 5}}, \gamma_{2}>\gamma_{1} \text { and } \gamma=\left(\frac{N}{g}\right)^{1 / 2} \text { the }
$$

number of monomers per adsorbed chain on the surface is $N_{\mathrm{S}} \sim \frac{b^{D}}{N^{1 / 2} \phi_{\mathrm{b}}^{5 / 8}}$

(iii) when $b$ is larger than the radius $R\left(\phi_{\mathrm{b}}\right)$, we may use the results obtained for an infinite surface.

\section{Discussion.}

Our results for the adsorbance are summarized in figure 2 where the various behaviours are displayed in a plane bulk concentration $\phi_{\mathrm{b}}$-grain radius $b$. Three main regions should be distinguished.

At very small radius $b$ only one chain adsorbs [15]. This chains builds up a diffuse layer whose thickness is comparable to the grain radius $b$. A single polymer chain adsorbing on a plane forms a flat pancake with a thickness of the order of the monomer length $a$. On an infinite surface, energy is gained by putting more and more monomers in contact with the surface and no loops are formed. On very small colloidal grains the surface is saturated when $N^{*}\left(\sim b^{D}\right)$ monomers are adsorbed, the free energy gain is in any case $\sim N^{*} T$. The entropy is increased by forming small loops up to a size $b$. In a good solvent large loops are unlikely, their distribution is however very broad and they contribute to the concentration profile.

For small concentrations and large radii, the adsorbance is equal to the surface excess ; the total number of adsorbed chains is proportional to the available surface $\left(\frac{b^{D}}{N}\right)$ each adsorbed chain has $N_{\mathrm{S}} \sim N$ monomers in contact with the surface. 


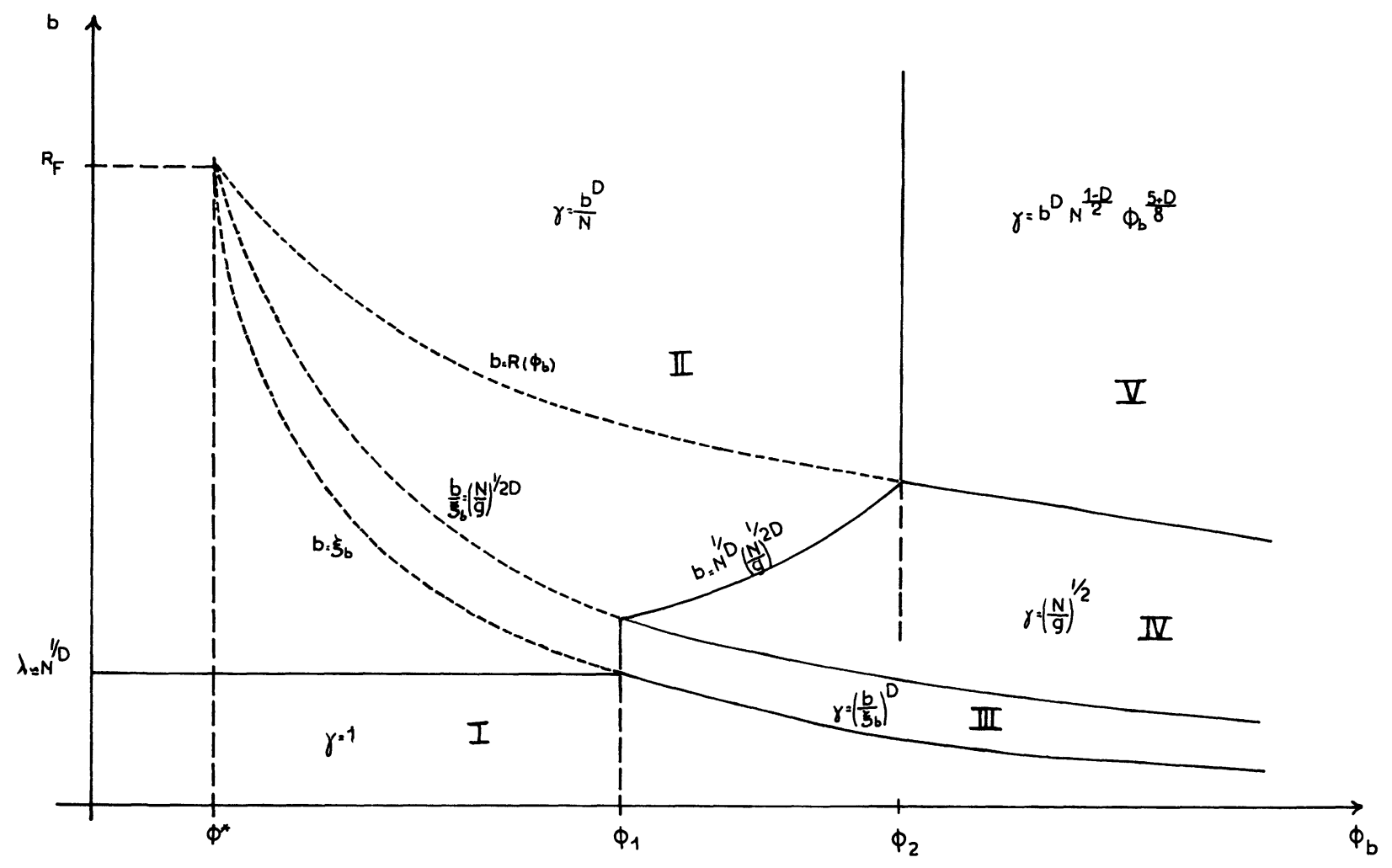

Fig. 2. - Various regimes for the adsorbance of a semi-dilute polymer solution in contact with a fractal colloidal grain in a plane grain size $b$-concentration $\phi_{\mathrm{b}}$. The crossover full lines divide the plane in 5 regimes : in region $\mathrm{I}$; only one chain is adsorbed ; in region II ; the number of adsorbed chain $\gamma$ is proportional to the available surface $\gamma \sim \frac{b^{D}}{N}$; in region III, IV, V, $\gamma$ depends on the bulk concentration : $\gamma=\left(\frac{b}{\xi}\right)^{D}$ region III ; $\gamma=\left(\frac{N}{g}\right)^{1 / 2}$ region IV; $\gamma=b^{D} N^{\frac{1-D}{2}} \phi_{\mathrm{b}}^{\frac{5+D}{8}}$ region $\mathrm{V}$.

For larger concentrations the semi-dilute solution may be viewed as a melt of blobs and three different regimes are found according to the relative value of the grain radius and the chain radius. The crossovers between these regimes are presented here as sharp but one should keep in mind that this is just a scaling theory and that they are probably much smoother in practice. As already noted by De Gennes [16], a B.E.T. measurement of the adsorbance as a function of the polymer radius does not give a determination of the surface fractal dimension but rather of the polymer fractal dimension in a dilute solution. In a concentrated solution, the solid fractal dimension could be obtained in certain regimes by varying the bulk concentration or the polymer mass.

We have studied adsorption on small colloidal grains but we expect these results to be valid for adsorption on any kind of surface with a restricted geometry. An example of such surfaces is an infinite surface repulsive for the polymer with a small adsorbing spot of size $b$. This is thus a first step towards the understanding of polymer adsorption on heterogeneous surfaces; a needed extension of this problem is then the adsorption on a repulsive surface with two adsorbing spots (or equivalently the adsorption on two spherical grains at a given distance). The extreme limit of the interaction between a semidilute solution and a small but finite concentration of spheres has been studied theoretically by Alexander [17] and experimentally by Cabane and his coworkers. In certain concentration ranges for the spheres and the polymer, a gel is formed where the spheres (small micelles) adsorbing the polymer act as crosslinks between different chains. The micelle radius is always smaller than the correlation length $\xi_{\mathrm{b}}$ and we predict a number of adsorbed chains per micelle of order 1 . Our scaling theory is not thus refined enough to discuss gel formation in these systems. 


\section{References}

[1] Cohen Stuart, M., Cosgrove, T., Vincent, B., Adv. Colloid Interface Sci. 24 (1986) 143.

[2] De Gennes, G., Adv. Colloid Interface Sci. 27 (1987) 189.

[3] Ober, R., Paz, L., Taupin, C., Pincus, P., BolleaU, S., Macromolecules 16 (1983) 50.

[4] Kawaguchi, M., Takahashi, A., Macromolecules 16 (1983) 1465.

[5] Auvray, L., Cotton, J. P., Macromolecules 20 (1987) 202.

[6] Cabane, B., Duplessix, R., J. Phys. France 48 (1987) 651.

[7] Eisenriegler, E., Kremer, K., Binder, K., J. Chem. Phys. 77 (1982) 6296.

[8] De Gennes, P. G., Macromolecules 14 (1981) 1637.

[9] DE GenNes, P. G., Scaling concepts in polymer physics (Cornell University Press, second printing) 1985 .
[10] Binder, K., Kremer, K., Scaling phenomena in disordered systems, Eds. R. Pynn, A. Skjeltrop (Plenum New York) 1985.

[11] MANDELBRoJT, B., The fractal geometry of nature (Freeman San Francisco) 1982.

[12] De Gennes, P. G., C.R. Hebd. Séan. Acad. Sci. 299 II (1984) 913.

[13] De Gennes, P. G.; C.R. Hebd. Séan. Acad. Sci. $292 I$ (1981) 701.

[14] De Gennes, P. G., C.R. Hebd. Séan. Acad. Sci. 294II (1982) 1317.

[15] The adsorption of isolated polymer chains has been discussed by PINCUS, P., SANDrofF, C., WITTEN, T., J. Phys. Lett. France 45 (1984) 725.

[16] See reference [12].

[17] Alexander, S., J. Phys. France 38 (1977) 977. 